\title{
The clinical course of SARS-CoV-2 infection among children with rheumatic disease under biologic therapy: a retrospective and multicenter study
}

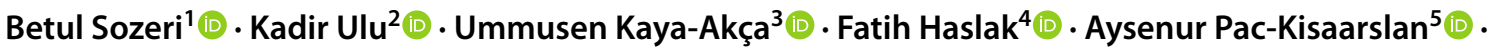

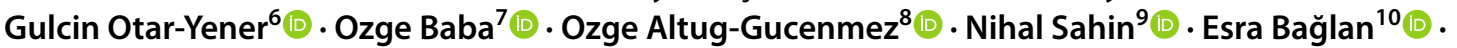

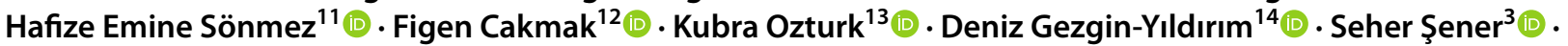

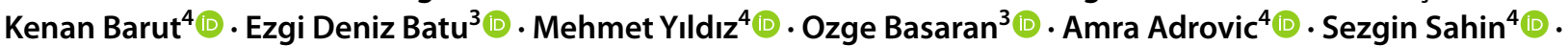

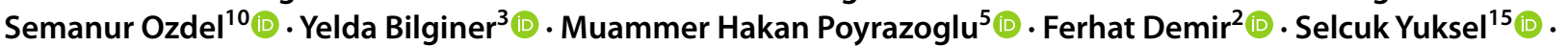 \\ Mukaddes Kalyoncu ${ }^{7}$ (D) Ozgur Kasapcopur ${ }^{4}$ (D) Seza Ozen $^{3}$ (D) . Nuray Aktay-Ayaz ${ }^{12}$ (])
}

Received: 2 August 2021 / Accepted: 20 September 2021 / Published online: 27 September 2021

(๑) The Author(s), under exclusive licence to Springer-Verlag GmbH Germany, part of Springer Nature 2021

\begin{abstract}
The effects of biological disease-modifying antirheumatic drugs (bDMARDs) in the clinical course of COVID-19 on children with underlying rheumatologic diseases have not been fully demonstrated. To evaluate the course of COVID-19 infection in patients with rheumatic disease receiving bDMARD treatment. This was a retrospective, multicenter study conducted in pediatric patients infected by SARS-CoV-2 and under bDMARDs therapy. The study population consisted of 113 patients (72 female/41 male). The mean age of the patients was $12.87 \pm 4.69$ years. The primary diagnosis of the cohort was as follows: 63 juvenile idiopathic arthritis, 35 systemic autoinflammatory diseases, 10 vasculitides, and five cases of connective tissue diseases. The mean duration of the primary disease was $4.62 \pm 3.65$ years. A total of 19 patients had additional comorbid diseases. Thirty-five patients were treated with canakinumab, 25 with adalimumab, 18 with etanercept, 10 with infliximab, nine with tocilizumab, six with rituximab, four with anakinra, three with tofacitinib, and one with abatacept. The median exposure time of the biological drug was 13.5 months. Seventy-one patients had symptomatic COVID-19, while 42 were asymptomatic. Twenty-four patients required hospitalization. Five patients presented with MIS-C. The hospitalized patients were younger and had a shorter duration of rheumatic disease compared to ambulatory patients, although the difference was not statistically significant. Steroid usage, presence of fever, and dyspnea were more common among the hospitalized patients. A worsening in the course of both COVID-19 and current disease was not noticed under bDMARDs, however, to end with a strong conclusion multicentric international studies are required.
\end{abstract}

Keywords COVID-19 $\cdot$ Rheumatic disease $\cdot$ Biologic drugs $\cdot$ Pediatrics

\section{Introduction}

Coronavirus disease 19 (COVID-19) has been the foremost cause of morbidity and mortality in the world during the past 2 years. Initially, a cluster of unexplained pneumonia was reported from Wuhan, Hubei province, China in December

Congress abstract publications: This data was accepted as a poster in. 27th European Pediatric Rheumatology Congress.

Betul Sozeri

drbetulsozeri@gmail.com

Extended author information available on the last page of the article
2019. Subsequently, a new type of coronavirus named SARS-CoV-2 was found to be the causative viral agent; this new disease was declared as COVID-19 on February 11, $2020[1,2]$. Until date (accepted date: September 3, 2021), 218.9 million confirmed cases of COVID-19 have been reported, of which 4.5 million confirmed death have been reported globally by the World Health Organization (WHO). In our country, 6.4 million infected cases and 57.283 cumulative deaths have been announced in the same time period [3]. The most common symptoms during the SARS-CoV-2 infection are fever, cough, shortness of breath, fatigue, myalgia, loss of taste or smell, and sore throat. However, it is well-known now that COVID-19 is clinically characterized 
by a varying spectrum, ranging from an asymptomatic course to life-threatening multi-organ failure [1, 2]. Initial reports showed that children with COVID-19 were prone to develop a mild or asymptomatic course. However, children with a rheumatic disease or those receiving immunosuppressant treatments may be vulnerable to SARS-CoV-2 infection.

It is well-known that patients with rheumatic disease are at risk of serious infection due to both immune dysfunctions resulting from their diseases and the usage of immunomodulatory drugs. Biological disease-modifying anti-rheumatic drugs (bDMARDs), which are often used in treatments, act by targeting the pathways of the immune system through cytokine blockade and can cause immune dysregulation in the body. Increased risk of serious infections in both adults and children has been reported with the use of bDMARDs [4]. Therefore, the presence of a rheumatic disease or the usage of anti-rheumatic drugs is of interest in terms of whether or not they affect the course of SARS-CoV-2 infection. A meta-analysis by Akiyama et al. [5] showed that having a rheumatic disease increased the risk of the symptomatic course of COVID-19, rate of hospital admission, and death due to COVID-19 in adult patients when compared to that in the general population. On the other hand, conflicting results have been reported that suggest that patients with rheumatic diseases and SARS-CoV-2 express milder symptoms of COVID-19 without any severe complications due to the usage of corticosteroids and bDMARDs [6]. Although the number of studies published on COVID-19 is increasing every day, the data on pediatric patients remain insufficient. Recently, a study from Italy [7] concluded that pediatric patients with chronic diseases who were treated with bDMARDs did not carry an increased risk for respiratory or life-threatening complications of COVID-19 relative to the general population. Restricted reports with a small number of patients $[8,9]$ have been introduced from our country; however, relevant multicentric data from developing countries are still lacking.

Herein, we aimed to determine the course of SARSCoV-2 infection in children with rheumatic diseases under bDMARD treatment.

\section{Methods}

This retrospective, multicenter study was conducted by 14 pediatric rheumatology centers in Turkey. The medical records of patients who were diagnosed with a rheumatic disease according to the previously accepted criteria and treated with bDMARDs were reviewed.

\section{Inclusion and exclusion criteria}

Patients with rheumatic diseases and receiving bDMARDs were evaluated, and of these, the patients who were diagnosed with COVID-19 were included in the study. Case definition of COVID-19 was made according to the following criteria:

a) In asymptomatic patient, the detection of SARS-CoV-2 RNA was performed using molecular amplification test [e.g., nasal polymerase chain reaction (PCR) test].

b) In asymptomatic patients, the detection of SARS-CoV-2 RNA was performed using PCR. The patient had a history of contact with a confirmed case.

c) In patients, the detection of positive SARS-CoV-2 antibody [immunoglobulin (Ig) $\mathrm{M}$ and IgG] ELISA test after suspected COVID-19 symptoms, but without SARS-CoV-2 PCR confirmation during the symptomatic period.

d) In patients, the detection of SARS-CoV-2 antibody IgM and $\mathrm{IgG}$ after a close contact with a person infected with SARS-CoV-2.

The diagnosis of COVID-19 was confirmed in 103 patients by nasal SARS-CoV-2 PCR and in 10 patients by SARS-CoV-2 antibody IgM and IgG ELISA test.

Patients infected with SARS-CoV-2, but not receiving bDMARDs were excluded from the study. Furthermore, patients who had suspected symptoms, but refused to undertake PCR or antibody test for confirmation of the diagnosis were excluded.

\section{Data collection}

This real-life study was based on secondary data collection from medical records of patients between April 2020 and 2021. Demographic and clinical data, laboratory findings, information about the course of COVID-19, and the outcomes were retrospectively recorded.

The present study was approved by the Institutional Review Board (IRB) at xxxx via waiver of informed consent (IRB number STUDY00000771).

\section{Statistical analysis}

The SPSS version 21.0 (SPSS, Inc., Chicago, Illinois) was used for statistical analysis. The variables were investigated using visual (histogram, probability plots) and analytic methods (Kolmogorov-Smirnov/Shapiro-Wilk's test) to determine whether or not they were normally distributed. Descriptive analyses were presented using the proportion 
for categorical variables; mean and standard deviation (SD) were used for normally distributed numerical variables; and median and range (R) were used for non-normally distributed numerical variables. Differences in the proportions between groups were compared by Chi-squared test or Fisher's exact test, whichever was appropriate. The Student's $t$ test (for normally distributed variables) or the Mann-Whitney $U$ test (for non-normally distributed variables) was performed to evaluate the differences between the two groups when comparing numerical data. $P \leq 0.05$ was considered to indicate statistical significance.

\section{Results}

\section{Baseline characteristics of the patient group}

We included 113 pediatric patients infected with SARSCoV-2 who were receiving bDMARD for the treatment of their primary rheumatic diseases. The study population consisted of 72 female (63.7\%) and 41 (36.3\%) male. The mean age of the patients was $12.87 \pm 4.69$ years. Among 113 patients, 63 (55.8\%) were diagnosed with juvenile idiopathic arthritis (JIA), 35 (30.9\%) with systemic autoinflammatory disease (AID), 10 (8.9\%) with vasculitides, and 5 (4.4\%) with connective tissue diseases. The mean duration of the primary rheumatic disease was $4.62 \pm 3.65$ years. Nineteen (16.8\%) patients had additional comorbid diseases (Table 1).

Prior to SARS-CoV-2 infection, 35 patients (30.9\%) were receiving canakinumab, $26(23.0 \%)$ were treated with adalimumab, $19(16.8 \%)$ with etanercept, 10 (8.9\%) with infliximab, $9(8.0 \%)$ with tocilizumab, $6(5.3 \%)$ with rituximab, 4 (3.5\%) with anakinra, 3 (2.7\%) with tofacitinib, and $1(0.9 \%)$ with abatacept. Furthermore, 42 (37.1\%) patients were using conventional DMARD, concomitantly and 27 (23.8\%) patients were also receiving corticosteroids with a median dose of $0.16 \mathrm{mg} / \mathrm{kg}$ (Table 1). The median time of exposure to a biological drug was 12 months. The median time of duration between diagnosis of COVID-19 and the last biologic drug administration was 15 days.

\section{COVID-19 test results}

In the cohort, 101 (89.4\%) patients had a history of close contact with COVID-19 patients. Among them, 18 patients had an infected person in their family. At the time of diagnosis, SARS-CoV-2 PCR was positive in 103 (91.1\%) patients. The diagnosis of COVID-19 was confirmed by SARS-CoV-2 antibody test in the remaining 10 patients (8.9\%). Of these 10 patients, all had COVID-19-suspected symptoms, but SARS-CoV-2 PCR test was not performed when they were symptomatic.
Table 1 Baseline characteristics of the patients infected with SARS$\mathrm{CoV}-2$ while receiving a bDMARD

\begin{tabular}{|c|c|}
\hline \multicolumn{2}{|l|}{ Rheumatic disease, $n(\%)$} \\
\hline Juvenile idiopathic arthritis & $63(55.8 \%)$ \\
\hline Autoinflammatory diseases & $35(30.9 \%)$ \\
\hline Familial Mediterranean fever & 21 \\
\hline Cryopyrin associated periodic syndrome & 5 \\
\hline Hyperimmunoglobulin D syndrome & 4 \\
\hline Chronic recurrent osteomyelitis & 3 \\
\hline Idiopathic recurrent pericarditis & 1 \\
\hline $\begin{array}{l}\text { Pyogenic arthritis, pyoderma gangrenosum and acne } \\
\text { syndrome }\end{array}$ & 1 \\
\hline Vasculitis & $10(8.9 \%)$ \\
\hline Adenosine deaminase 2 deficiency & 4 \\
\hline Takayasu arteritis & 3 \\
\hline Polyarteritis nodosa & 1 \\
\hline Other & 2 \\
\hline Connective tissue diseases & $5(4.4 \%)$ \\
\hline Systemic lupus erythematosus & 3 \\
\hline Sjögren's disease & 1 \\
\hline Juvenile dermatomyositis & 1 \\
\hline Comorbidities, $n(\%)$ & $19(16.8 \%)$ \\
\hline Hypertension & 4 \\
\hline Inflammatory bowel disease & 3 \\
\hline Scoliosis & 3 \\
\hline Chronic renal failure & 2 \\
\hline Hereditary spherocytosis & 1 \\
\hline Asthma & 1 \\
\hline Cardiomyopathy & 1 \\
\hline Adrenal insufficiency & 1 \\
\hline Celiac disease & 1 \\
\hline Growth hormone insufficiency & 1 \\
\hline Hypothyroidism & \\
\hline Disease-modifying antirheumatic drugs, $n(\%)$ & $42(37.1 \%)$ \\
\hline Methotrexate & 27 \\
\hline Mycophenolate mofetil & 5 \\
\hline Leflunomide & 4 \\
\hline Azathioprine & 3 \\
\hline Cyclosporine & 1 \\
\hline Sulfasalazine & 1 \\
\hline Hydroxychloroquine & 1 \\
\hline
\end{tabular}

\section{The clinical course of patients during the SARS-CoV-2 infection}

At the time of diagnosis of COVID-19, 71 (62.8\%) patients had at least one COVID-19-related symptom, while 42 $(37.2 \%)$ were asymptomatic. The most common symptom was fever $(n=42)$, followed by myalgia $(n=34)$ and cough $(n=29)$ (Table 2). The median duration of fever was 4 (1-16) days. Twenty-nine patients (25.6\%) presented with respiratory symptoms such as cough and dyspnea. Abnormal 
Table 2 Clinical findings of the patients at the time of diagnosis of COVID-19

\begin{tabular}{ll}
\hline Asymptomatic, $n(\%)$ & $42(37.2 \%)$ \\
Symptomatic, $n(\%)$ & $71(62.8 \%)$ \\
Fever & 42 \\
Myalgia & 34 \\
Cough & 29 \\
Anosmia/ageusia & 15 \\
Diarrhea & 11 \\
Abdominal pain & 9 \\
Dyspnea & 8 \\
Rash & 5 \\
Anorexia/nausea/emesis & 4 \\
Fatigue/malaise & 3 \\
Chest pain & 3 \\
\hline
\end{tabular}

thorax computerized tomography findings revealed COVID19 in $7(6.2 \%)$ patients.

Twenty-four $(21.2 \%)$ patients required hospitalization. The median time of hospitalization was 8 (2-10) days. Of these 24 patients, two were admitted to the intensive care unit with the requirement of mechanical ventilation. The remaining 89 (78.8\%) patients received ambulatory care. Treatment was applied to $35(30.9 \%)$ patients. Among these 35 patients, 21 were hospitalized. Twenty-two received favipiravir, 10 received azithromycin, and seven received oseltamivir. Ten patients required steroid treatment, concomitantly.

Five $(4.4 \%)$ patients of whom two with the diagnosis of JIA, two with vasculitis, and one with AID presented with MIS-C. These two patients with vasculitis were receiving anti-tumor necrosis factor (TNF)- $\alpha$ drugs (etanercept $=1$, infliximab $=1)$. Two patients with JIA were using antiinterleukin (IL)-1 agents (canakinumab = 1, anakinra =1). The patient with AID was receiving canakinumab. Among these five patients, four were treated with IVIG and anakinra, two patients also received tocilizumab. Two patients died. Of these two patients, one was diagnosed with vasculitis and treated with etanercept before MIS-C, and the other was diagnosed with JIA and was receiving anakinra.

None of the patients reported disease flare after COVID19 or the occurrence of any additional late complication after post-COVID-19 during the follow-up.

\section{Comparison of clinical findings of patients requiring hospitalization with others}

The comparison of clinical and laboratory findings of patients requiring hospitalization and receiving ambulatory care are depicted in Table 3. No differences were noted in terms of the type of rheumatic diseases or biological drugs used as well as the median duration time of biologic drug administration. Patients requiring hospitalization were younger and had a shorter duration of rheumatic disease when compared to others, while these findings did not reach any statistical significance. Furthermore, steroid usage was more common among patients requiring hospitalization.

On evaluation, the risk factor associated with hospitalization, the presence of fever [OR 3.05 (95\% CI 1-7.7), $P=0.01$ ], and dyspnea [OR 4. 25 (95\% CI 0.97-18.46), $P=0.04]$ increased the odds of hospitalization. However, not statistically different, patients treated with rituximab and tofacitinib were hospitalized for a longer time (Table 3).

\section{Discussion}

Immunomodulatory drugs or underlying diseases may influence the clinical course of SARS-CoV-2 infection. However, it remains debatable whether medications worsen or improve the symptoms of COVID-19 and the clinical outcomes. Furthermore, the effect of SARS-CoV-2 infection on the disease activity of the underlying rheumatic disease remains unclear. We believe that clarifying these issues will guide clinicians when dealing with rheumatic diseases. This multicenter study presents the clinical characteristics and outcomes of patients infected with SARS-CoV-2 who were receiving bDMARD due to their primary rheumatic diseases. The overall hospitalization rate was $21.2 \%$, while $78.8 \%$ of the patients received ambulatory care. The presence of fever and dyspnea were found to be risk factors for hospitalization. Hospitalized patients had younger age, shorter disease duration, and a higher rate of steroid usage when compared to ambulatory patients.

It is well-known that patients with comorbidities such as cardiovascular disease, liver disease, kidney disease, or malignancies are more vulnerable to SARS-CoV-2 infection [10, 11]. Correspondingly, a large pediatric cohort from our country revealed an association between having an underlying disease and a severe course of COVID-19 [12]. However, the course of COVID-19 in patients with a rheumatic disease or those using antirheumatic drugs remains unclear. Hyrich et al. [6] reported an increased risk of death among patients with rheumatic arthritis (RA), systemic lupus erythematosus, and psoriasis when infected with SARS-CoV-2. Gianfrancesco et al. [13] found that $10 \mathrm{mg} /$ day or a higher dose of glucocorticoid exposure increased the risk of hospitalization, while the hospitalization rate was lower in patients using anti-TNF. By the same group, it was also shown that patients with RA receiving rituximab or Janus kinase inhibitors presented with a more severe course of COVID-19 than those treated with anti-TNF [14]. Haberman et al. [15] confirmed that COVID-19 outcomes were worse in patients with inflammatory arthritis receiving glucocorticoids, but it did not differ in patients using anti-cytokine therapy. The data 
Table 3 The comparison of clinical findings of patients with requiring hospitalization and others

\begin{tabular}{|c|c|c|c|}
\hline & $\begin{array}{l}\text { Patients who were } \\
\text { ambulatory }(n=89)\end{array}$ & $\begin{array}{l}\text { Patients who were hospi- } \\
\text { talized }(n=24)\end{array}$ & $P$ value \\
\hline Female/male, $n$ & $57 / 32$ & $15 / 9$ & 0.53 \\
\hline The mean of age (years) & 14.4 & 11.5 & 0.44 \\
\hline Distribution of rheumatic disease, $n$ & & & 0.27 \\
\hline Juvenile idiopathic arthritis & 51 & 12 & \\
\hline Systemic autoinflammatory diseases & 29 & 6 & \\
\hline Vasculitis & 7 & 3 & \\
\hline Connective tissue diseases & 2 & 3 & \\
\hline $\begin{array}{l}\text { The mean } \pm \text { SD duration of primary rheu- } \\
\text { matic disease (months) }\end{array}$ & $41.5 \pm 43.5$ & $35.2 \pm 45.04$ & 0.30 \\
\hline Comorbid disease, $n$ & 14 & 5 & 0.42 \\
\hline Usage of corticosteroids, $n$ & 17 & 10 & $\mathbf{0 . 0 3}$ \\
\hline Median dose of steroid, $\mathrm{mg} / \mathrm{kg}$ & $0.16(0.05-2)$ & $0.15(0.02-0.6)$ & 0.34 \\
\hline Usage of DMARD, $n$ & 38 & 8 & 0.27 \\
\hline Distribution of biologic drugs, $n$ & & & 0.33 \\
\hline Canakinumab & 26 & 9 & \\
\hline Infliximab & 9 & 1 & \\
\hline Adalimumab & 22 & 4 & \\
\hline Etanercept & 16 & 3 & \\
\hline Tocilizumab & 8 & 1 & \\
\hline Anakinra & 2 & 2 & \\
\hline Rituximab & 4 & 2 & \\
\hline Abatacept & 1 & 0 & \\
\hline Tofacitinib & 1 & 2 & \\
\hline $\begin{array}{l}\text { The median exposure time of a biologic } \\
\text { drug (months) }\end{array}$ & $12.8(1-95)$ & $13.0(1-84)$ & 0.65 \\
\hline Fever, $n$ & 14 & 28 & 0.01 \\
\hline Myalgia, $n$ & 25 & 9 & 0.37 \\
\hline Cough, $n$ & 19 & 10 & 0.06 \\
\hline Anosmia/ageusia, $n$ & 15 & 0 & 0.03 \\
\hline Diarrhea, $n$ & 9 & 2 & 0.79 \\
\hline Abdominal pain, $n$ & 7 & 2 & 0.94 \\
\hline Dyspnea, $n$ & 4 & 4 & 0.04 \\
\hline Rash, $n$ & 2 & 3 & 0.06 \\
\hline Anorexia/nausea/emesis, $n$ & 3 & 1 & 0.62 \\
\hline
\end{tabular}

The statistical significant parameters were presented in bold about the clinical course of pediatric patients infected with SARS-CoV-2 who were receiving bDMARD is limited. A study from Turkey evaluated 39 children with a rheumatic disease who were infected with SARS-CoV-2 while receiving biological drugs. Among these 39 patients, 21 were symptomatic and 18 were asymptomatic. The hospitalization rate was $51.3 \%$, and the mortality rate was $2.5 \%$ [8]. Villacis-Nunez et al. [7] reported 55 cases with rheumatic diseases and laboratory-confirmed COVID-19. In this cohort, the hospitalization rate was $18.2 \%$. Afro-American ethnicity, presence of cardiovascular diseases, active rheumatic disease, and the usage of medium/high-dose corticosteroid or mycophenolate or rituximab were found to be associated with an increased odds ratio of hospitalization.
Furthermore, fever, dyspnea, chest pain, and rash were more common among the hospitalized patients. Correspondingly, we showed that steroid usage, the presence of fever, and dyspnea were more common among the hospitalized patients. However, we could not demonstrate any differences between hospitalized patients and ambulatory patients in terms of rheumatic disease diagnosis or the type of antirheumatic drugs received or comorbidities. In addition, we found that patients treated with rituximab and tofacitinib were hospitalized for a longer time. Most recently, a nationwide registry of biologics in pediatric rheumatology from Germany presented the outcomes of 76 patients with a rheumatic disease and laboratory-confirmed SARS-CoV-2 infection [16]. Among these patients, $76 \%$ patients were treated 
with DMARDs, $41 \%$ with biologic drugs, and $11 \%$ with systemic corticosteroids. They concluded that children with rheumatic diseases under various medications had a milder course of SARS-CoV-2 infection with favorable outcomes. On the same lines, they did not demonstrate any association between the underlying disease activity and the course of COVID-19 [16].

The primary disease may deteriorate further during the SARS-CoV-2 infection. Disease flare was reported by previous reports in children infected with SARS-CoV-2 who were receiving bDMARD $[6,13]$. However, we could not demonstrate disease flare after COVID-19 or an additional late complication after post-COVID-19 during follow-up.

During the SARS-CoV-2 infection, aberrant immune response plays a central role, especially in severe cases, and it is well-known that, during childhood, both the morbidity and mortality of COVID-19 are commonly related to MIS-C rather than to primary SARS-CoV-2 infection. Villacis-Nunez et al. [7] did not describe an MIS-C patient in their cohort of 55 pediatric patients with rheumatic diseases. Interestingly, five of the 113 cases developed MIS-C after asymptomatic COVID-19. All of them required hospitalization and two of them died. While this association has not been elucidated, the pattern of immune reaction in primary rheumatic disease may influence the response to SARS-CoV-2.

The retrospective design of this study and the small number of patients limited our results, but the multicenter design strengthened our study. As the ethnicity and socioeconomic status may influence the course of infectious diseases, our results are quite important with clinical characteristics and the outcomes of patients from a developing country infected with SARS-CoV-2 while receiving a bDMARD. Moreover, the small sample size did not allow more detailed statistical analysis and presented the risk factors for hospitalization and severe course completely.

\section{Conclusion}

In patients with underlying comorbidities, COVID-19 may have a severe course, regardless of the use of bDMARD. However, in the present study, we could not demonstrate whether the course of COVID-19 or the primary disease worsened in patients receiving bDMARDs. Further multicenter, international studies are believed to demonstrate the risk factors for a severe course of COVID-19 among patients with rheumatic diseases.

Author contributions BS conceptualized and designed the study, drafted the initial manuscript, and had full access to all the data in the study. All authors conducted the data analyses, drafted the initial manuscript, and had full access to all the data in the study. All authors reviewed and revised the manuscript and approved the final version of the manuscript.

Funding No source of funding for this work.

Availability of data and material Not applicable.

Code availability Not applicable.

\section{Declarations}

Conflict of interest None of the authors received financial support and there are no potential conflicts of interest.

Ethics approval The present study was approved by the Institutional Review Board (IRB) at Umraniye Training and Research Hospital via waiver of informed consent (IRB number STUDY00000771).

\section{References}

1. World Health Organization. Director-General's remarks at the media briefing on 2019-nCoV on 11 February 2020. http://www. who.int/dg/speeches/detail/who-director-general-s-remarks-atthe-media-briefing-on-2019-ncov-on-11-february-2020 (Accessed on February 12, 2020)

2. World Health Organization (WHO). WHO Director-General's opening remarks at the media briefing on COVID-19-11 March 2020. Available at: https://www.who.int/dg/speeches/detail/whodirector-general-s-opening-remarks-at-the-media-briefing-oncovid-19-11-march-2020 (Accessed on May 01, 2020)

3. World Health Organization https://www.who.int/emergencies/ diseases/novel-coronavirus-2019

4. Swart J, Giancane G, Horneff G, Magnusson B, Hofer M, Alexeeva $E$ et al (2018) Pharmacovigilance in juvenile idiopathic arthritis patients treated with biologic or synthetic drugs: combined data of more than 15,000 patients from pharmachild and national registries. Arthritis Res Ther 20:285

5. Akiyama S, Hamdeh S, Micic D, Sakuraba A (2021) Prevalence and clinical outcomes of COVID-19 in patients with autoimmune diseases: a systematic review and meta-analysis. Ann Rheum Dis 80:384-391

6. Hyrich KL, Machado PM (2021) Rheumatic disease and COVID19: epidemiology and outcomes. Nat Rev Rheumatol 17:71-72

7. Villacis-Nunez DS, Rostad CA, Rouster-Stevens K, Khosroshahi A, Chandrakasan S, Prahalad S (2021) Outcomes of COVID-19 in a cohort of pediatric patients with rheumatic diseases. Pediatr Rheumatol Online J 19:94

8. Demir F, Ulu K, Çağlayan Ç, Coşkuner T, Sözeri S (2021) Clinical course of COVID-19 in children with rheumatic disease under biologic therapy. Clin Exp Rheumatol 128:36-37

9. Aydın F, Kurt T, Sezer M, Tekgöz N, Tekin ZE, Yüksek SK et al (2021) Biological therapies in children with rheumatic diseases during the COVID-19 pandemic: a single-centre experience. Int J Clin Pract 75:e14030

10. Zhou F, Yu T, Du R, Fan G, Liu Y, Liu Z et al (2020) Clinical course and risk factors for mortality of adult inpatients with COVID-19 in Wuhan, China: a retrospective cohort study. Lancet 395:1054-1062 
11. Wang T, Du Z, Zhu F, Cao Z, An Y, Gao Y et al (2020) Comorbidities and multi-organ injuries in the treatment of COVID-19. Lancet 395:52

12. Karbuz A, Akkoc G, Bedir Demirdag T, Yilmaz Ciftdogan D, Ozer A, Cakir D et al (2021) Epidemiological, clinical, and laboratory features of children with COVID-19 in Turkey. Front Pediatr 9:631547

13. Gianfrancesco M, Hyrich KL, Al-Adely S, Carmona L, Danila MI, Gossec L et al (2020) Characteristics associated with hospitalization for COVID-19 in people with rheumatic disease: data from the COVID-19 global rheumatology alliance physician-reported registry. Ann Rheum Dis 79:859-866

14. Sparks JA, Wallace ZS, Seet AM, Gianfrancesco MA, Izadi Z, Hyrich KL et al (2021) Associations of baseline use of biologic or targeted synthetic DMARDs with COVID-19 severity in rheumatoid arthritis: results from the COVID-19 global rheumatology alliance physician registry. Ann Rheum Dis 80:1137-1146
15. Haberman RH, Castillo R, Chen A, Yan D, Ramirez D, Sekar V et al (2020) COVID-19 in patients with inflammatory arthritis: a prospective study on the effects of comorbidities and diseasemodifying antirheumatic drugs on clinical outcomes. Arthritis Rheumatol 72:1981-1989

16. Sengler C, Eulert S, Minden K, Niewerth M, Horneff G, Kuemmerle-Deschner J et al (2021) Clinical manifestations and outcome of SARS-CoV-2 infections in children and adolescents with rheumatic musculoskeletal diseases: data from the national paediatric rheumatology database in Germany. RMD Open 7:001687

Publisher's Note Springer Nature remains neutral with regard to jurisdictional claims in published maps and institutional affiliations.

\section{Authors and Affiliations}

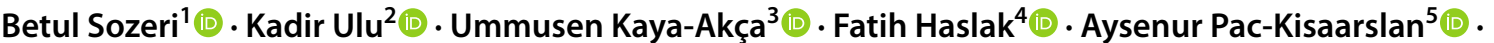

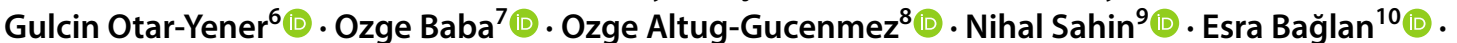

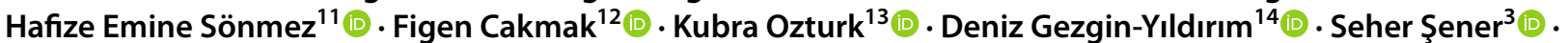

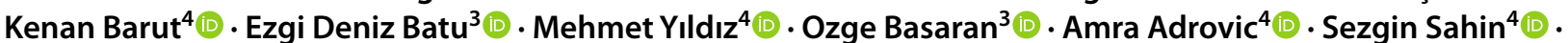

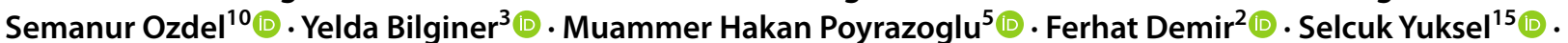 Mukaddes Kalyoncu $^{7}$ (1) $\cdot$ Ozgur Kasapcopur $^{4}$ (D) $\cdot$ Seza Ozen $^{3}$ (D) . Nuray Aktay-Ayaz ${ }^{12}$ (1)}

1 Department of Pediatric Rheumatology, University of Health Sciences, Ümraniye Research and Training Hospital, Istanbul, Turkey

2 Pediatric Rheumatology, University of Health Sciences, Umraniye Training and Research Hospital, Istanbul, Turkey

3 Pediatric Rheumatology, Hacettepe University Faculty of Medicine, Ankara, Turkey

4 Pediatric Rheumatology, Istanbul University-Cerrahpasa, Istanbul, Turkey

5 Pediatric Rheumatology, Erciyes University Faculty of Medicine, Kayseri, Turkey

6 Pediatric Rheumatology, Sanliurfa Training and Research Hospital, Sanliurfa, Turkey

7 Pediatric Rheumatology, Karadeniz Technical University Faculty of Medicine, Trabzon, Turkey

8 Health Sciences University, Dr Behcet Uz Children's Hospital, Izmir, Turkey
9 Pediatric Rheumatology, Health Sciences University, Bursa Center Hospital, Bursa, Turkey

10 Pediatric Rheumatology, Health Sciences University, Sami Ulus Training and Research Hospital, Ankara, Turkey

11 Pediatric Rheumatology, Kocaeli University Faculty of Medicine, Kocaeli, Turkey

12 Pediatric Rheumatology, Faculty of Medicine, Istanbul University, Istanbul, Turkey

13 Pediatric Rheumatology, Medeniyet University, Goztepe Training and Research Hospital, Istanbul, Turkey

14 Pediatric Rheumatology, Diyarbakır Children's Hospital, Diyarbakır, Turkey

15 Pediatric Rheumatology, Faculty of Medicine, Pamukkale University, Denizli, Turkey 